\title{
From the Editor's perspective
}

\author{
Theofanis Fotis PhD, MSc, BSc, RN
}

I $t$ is always difficult to share enthusiasm through the medium of paper. However, I would like to express 1 my excitement as being the new Co-Editor alongside Lucie Llewellyn. I am stepping into this new project following the legacy of the hard work of previous Editor Jessica Inch and Co-Editor Lee Lui. We have a responsibility to provide a high quality, scientifically oriented journal primarily for the members of British Anaesthetic and Recovery Nurses Association that also meets the needs of our international audience.

With regard to educational needs, the most important knowledge for a practicing nurse is the guidelines and research, which are being published by national and international institutes, associations and agencies. In everyday practice, it is crucial for nurses to use all the latest evidence from the available literature, evidence that is developed from trials, research and audit results. Over recent years, there have been an increasing number of nurses who are conducting research within their own fields of practice. Not only are they carrying out their own audits, but they are also accessing further education through attending university courses at different levels of higher education. What does this mean? It means that nurses in the United Kingdom and around the world have started producing evidence for their work, since they know exactly what is happening in their everyday clinical practice. They have a more detailed aspect of the needs, the problems, the mistakes and the deficiencies. As patient's advocates, they have a better knowledge than everyone else, what is best for these patients.

The reality is that everyday practice produces a huge amount of data that until recently, no one was collecting, analysing or publishing. This was not due to a lack of interest, but can be attributed in part to a lack of motivation, and in part to a lack of time. This journal cannot improve the amount of time available to carry out a study, but as a Co-Editor of this journal, I can commit that we will provide the space and as a result the motive for interested nurses to publish their work.

It is the time then, for clinical and academic nurses to start collecting the data that we produce, analyse and publish them. It is the time to develop the guidelines we need by ourselves and share them with the rest of the scientific world. Most hard-working nurses might have the opinion that all these guidelines and evidence are developed in academic offices or in scientific labs. Incorrect! All these results are coming from the analysis of the work of some hard-working nurses, maybe from the recovery department next door.

This is the reason why we have included in this issue a number of audits on different subjects from within practice. We are giving the opportunity to nurses to introduce their work, their measurements and to trigger a national and international exchange of knowledge and development of a common global quantitative perianaesthesia practice. The reader will find audits within this issue that have been completed in practice based on delayed discharge, staffing issues and anaesthetic handover.

Therefore, I address an open invitation to all our colleagues not only from within the United Kingdom, but also from abroad to contribute their work to the journal, giving them the opportunity to introduce their work to the international reading audience of the British Journal of Anaesthetic and Recovery Nursing. 